GRADUATE THESIS REPORT

\title{
Training of an intelligent agent to improve the gaming experience for video gamers
}

\author{
David Alejandro Alvarado Villa, Osval Antonio Montesinos López, Pedro C. \\ Santana-Mancilla
}

Published: 30 November 2021

\begin{abstract}
Today, video games have come a long way compared to the video games offered by the first consoles, both in technology and gameplay. However, one thing today's video games have in common with respect to the early video games is the use of intelligent agents to create a more competitive gaming environment for the video gamer. This work proposes the use of machine learning to create intelligent agents that analyze the player's skills and adjust their difficulty level according to these skills, with the aim of achieving a more immersive gaming experience and avoiding frustration for the video player.
\end{abstract}

\section{Keywords:}

Game experience; Dynamic complexity adjustment; Video games; Gameplay.

\section{Introducción}

En la historia de la humanidad han surgido diferentes actividades de ocio, que pueden ir desde leer, jugar a tres en raya (gato), pasear, hasta actividades que pueden involucrar cierto grado de destreza de la persona, como pintar o practicar algún deporte; estas son actividades donde la interacción es de una a dos personas o más dependiendo de la actividad.

Pero esto no era suficiente, conforme iba transcurriendo el tiempo se fueron desarrollando más actividades de ocio como los juegos de mesa, estos cuentan con diferentes reglas, con cantidad variable de jugadores, pero todos con el objetivo de pasar el rato jugando con más personas.

A la par las actividades lúdicas evolucionaban también las innovaciones relacionadas con la informática, hasta que llegó el momento donde convergieron los avances informáticos con las

Alvarado Villa, David Alejandro., Montesinos López, Osval Antonio., Santana-Mancilla, Pedro C.

Facultad de Telemática

Universidad de Colima

Av. Universidad 333

Colima 28040

dalvarado0@ucol.mx,oamontes1@ucol.mx,psantana@ucol.mx actividades de ocio, esto dio origen a uno de los conceptos con gran impacto hoy en día, los videojuegos. Uno de los primeros videojuegos fue Tennis for Two desarrollado en 1958 [2]. Ese videojuego partía del concepto del deporte de tenis, era un juego para dos personas con el objetivo de tener un mayor puntaje que el contrincante para ganar, conforme avanzaba la tecnología en el desarrollo de medios de almacenamiento como las memorias y los discos duros, esto dio la posibilidad de mejorar gráficamente los videojuegos además de contar con la posibilidad de procesar más información con los diferentes procesadores que fueron desarrollados en la década de los años 80 .

Los videojuegos también mejoraban en su manera de interactuar con los jugadores, pero la idea de jugar dos personas el mismo videojuego aún estaba lejos, esto dio paso a integrar oponentes generados por computadora con cierto grado de autonomía, como desplazarse por el escenario y ejecutar acciones simples, estos oponentes fueron los primeros intentos de simular los movimientos de una o más personas dentro de un juego de video, estos eran los primeros pasos de la inteligencia artificial en los videojuegos.

Un agente inteligente es un programa informático al cual se le asignan tareas ya sea por una persona u otro programa [5]. Para el desarrollo de las actividades, el agente inteligente requiere cierto grado de inteligencia, las características a destacar de los agentes son la comunicación ya sea entre un usuario, un programa u otros agentes, inteligencia que va de la mano con la complejidad de las tareas que se le vayan a asignar al agente, y autonomía que se enfoca a que el agente pueda trabajar sin supervisión.

\section{Motivación de la investigación}

Los avances tecnológicos han permitido crear videojuegos más elaborados gráficamente, videojuegos donde se tienen entornos virtuales de gran tamaño, en donde pasan una variedad de eventos en cada momento, con diferentes modos de juego, incluso jugar con otros jugadores a través del internet.

Hay videojuegos que se enfocan en la experiencia de jugador contra jugador, en donde los jugadores se sitúan en un entorno virtual y compiten para ser el ganador o estar en el grupo ganador, en esta categoría se encuentran los videojuegos de carreras, FPS (por sus siglas en inglés, First-Person Shooter), de peleas y los llamado Battle Royal, que en resumen consiste ser el último jugador o equipo en pie.

En muchas ocasiones no es posible contar con otras personas para jugar algún videojuego esto rompe la experiencia de juego tradicional, ya que un juego de carreras que se juegue descartando 
a otros jugadores humanos no tendría desafío alguno en ganar. Es por eso que el uso de agentes inteligentes que compitan contra los jugadores reales toma una importancia vital en la industria de los videojuegos.

Estos oponentes deben de ser regulados, ya que si son demasiados fáciles o difíciles el jugador puede perder el interés en seguir jugando, pero establecer ese nivel correcto no es tarea fácil, en muchos títulos de videojuegos, se le ofrece la oportunidad de elegir la dificultad, y se puede dividir de manera general en fácil, intermedio y difícil.

Contar con estos tres niveles predefinidos de dificultad, puede no ser suficiente para mantener enganchado al jugador. Es decir, dependiendo de la habilidad y experiencia del jugador, una dificultad predeterminada puede causar frustración tanto a jugadores avanzados como jugadores sin experiencia ya que no fueron diseñadas para adaptarse a la habilidad individual de cada uno.

Es en este punto, donde surge este trabajo de investigación, ya que existe una oportunidad de mejorar la experiencia de juego para las personas ajustando dinámicamente la dificultad en los videojuegos y de este modo personalizada a cada jugador. Para logarlo, se propone desarrollar un modelo de aprendizaje máquina que sea capaz de ajustarse automáticamente al nivel de experiencia mostrado por jugador.

\section{Trabajos relacionados}

El uso de agentes inteligentes en los videojuegos ha sido ampliamente estudiado. A continuación, se presentan algunas soluciones basadas en aprendizaje máquina para la creación de agentes inteligentes en videojuegos.

Perez-Liebana y colegas [4], presentaron un framework para la evaluación de agentes inteligentes y proveer a los investigadores de una plataforma de código abierto fácil de usar para probar sus métodos de inteligencia artificial con potencial de contar con juegos infinitos.

El juego presentado en [3] se trata de un videojuego de lucha que utiliza la técnica de árboles de búsqueda de Monte-Carlo (MCTS) para entrenar a sus agentes inteligentes para ser usados en juegos dentro de plataformas de transmisión en vivo (live streaming) como Twitch $(\mathrm{C}$ y YouTube $\mathbb{C}$.

El trabajo [6], presenta el desarrollo de un sistema de reconocimiento automático de frustración en los video jugadores. Esta propuesta puede servir como base para la evaluación de los agentes inteligentes que se desarrollen en el presente trabajo de investigación.

Con relación al reconocimiento de la atención que el video jugador está prestando al videojuego (si pierde la atención es posible que pierda el interés) se encuentra del trabajo [7], que utiliza retroalimentación neuronal para entrenar el modelo de reconocimiento de atención.

Diferentes trabajos abordan el desarrollo de agentes inteligentes para que puedan jugar ciertos juegos comerciales muy reconocidos y finalmente, algunos generan escenarios por medio de inteligencia artificial para hacer más difícil el juego como el presentado en [1].

En el análisis del estado del arte, se encontró que los diferentes trabajos que hay actualmente sobre el desarrollo de agentes inteligentes en videojuegos se enfocan principalmente en hacer que el agente juegue y complete algún videojuego. Es decir, no a que el agente inteligente controle las variables que dictan la dificultad del mismo videojuego, esto significa que el área de investigación sigue abierta y existe la posibilidad de realizar una contribución con este trabajo de tesis.

\section{$4 \quad$ Fase actual de investigación}

En la primera etapa del proyecto se realizó una investigación del estado del arte para identificar artículos y patentes relacionados al proyecto y compararlos para encontrar las oportunidades que este trabajo tiene, esto fue de ayuda para tener un punto de partida y tener un enfoque más preciso.

Actualmente se está en el proceso de análisis de herramientas para el entrenamiento del agente inteligente. Se tiene considerado usar OpenAI Gym. Se trata de un entorno de simulación el cual tiene como objetivo entrenar agentes inteligentes por medio de aprendizaje reforzado, el cual consiste en dar recompensas al agente durante su entrenamiento, de tal manera que todo el desarrollo se enfoca en la creación del agente inteligente, la idea de usar alguna herramienta como OpenAI Gym es aprovechar más el tiempo en el desarrollo del modelo que en el desarrollo de un videojuego.

Para lograrlo se proponen las capas mostradas en la Figura 1 y descritas a continuación.

- Jugador. Parte fundamental que va a interactuar con el sistema, el jugador puede ser cualquier persona que interactúa con el videojuego.

- Videojuego. Elemento con el que va a interactuar el jugador contra el agente inteligente. Este elemento se va a definir en la ejecución de la metodología, y será dentro del entorno virtual OpenAI Gym.

- Agente inteligente y dificultad. El agente inteligente a desarrollar depende directamente del videojuego al cual se va a incorporar, ya que las variables a considerar para el modelado, es decir, variables que afectan directamente a la jugabilidad de este, son diferentes dependiendo del tipo de videojuego, por lo tanto el cálculo de la dificultad es variable.

- Support Vector Machine. Es la técnica de entrenamiento que se va a usar en el modelado del agente inteligente.

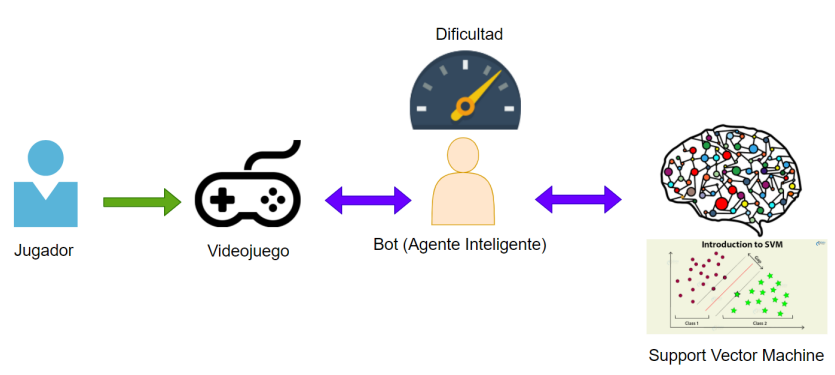

Figura 1.- Fases del Proyecto de investigación.

\section{Contribuciones esperadas y retos de la investigación}

Se espera que durante la ejecución de las fases que faltan en este trabajo de maestría se logren las siguientes contribuciones:

- Desarrollar un modelo de Aprendizaje Máquina que sea capaz de aprender del jugador para identificar su experiencia.

- Crear un modelo de Aprendizaje Máquina, para establecer dificultad dinámica del agente inteligente con base en la habilidad del jugador. 
- Implementar el agente inteligente en un videojuego, para que realice ajustes más precisos en la dificultad de acuerdo con el comportamiento del jugador.

\section{Motivación para asistir al consorcio de posgrado}

Asistir al consorcio de posgrado de MexIHC 2021 será enriquecedor para este trabajo, ya que se podrá obtener retroalimentación de expertos de reconocida trayectoria que guiarán los esfuerzos siguientes de esta investigación. Otra de las ventajas es conocer los trabajos de investigación que están realizando otros estudiantes de posgrado de las diferentes instituciones participantes y generar conexiones con ellos para posibles colaboraciones conjuntas.

\section{Referencias}

[1] Olve Drageset, Mark H. M. Winands, Raluca D. Gaina, and Diego Perez-Liebana. 2019. Optimising Level Generators for General Video Game AI. 2019 IEEE Conference on Games (CoG), IEEE, 1-8.

[2] William A Higinbotham. 2016. Tennis for Two. Stony Brook University. Retrieved from https://www.sunysb.edu/libspecial/videogames/tennis.ht $\mathrm{ml}$.

[3] Ryota Ishii, Suguru Ito, Ruck Thawonmas, and Tomohiro Harada. 2019. A Fighting Game AI Using Highlight Cues for Generation of Entertaining
Gameplay. 2019 IEEE Conference on Games (CoG), IEEE, $1-6$.

[4] Diego Perez-Liebana, Jialin Liu, Ahmed Khalifa, Raluca D. Gaina, Julian Togelius, and Simon M. Lucas. 2019. General Video Game AI: A Multitrack Framework for Evaluating Agents, Games, and Content Generation Algorithms. IEEE Transactions on Games 11, 3: 195-214.

[5] Marcela D Rodríguez, Pedro C Santana, Victor M González, Jesús Favela, and Ángel G Andrade. 2005. An Agent-based System to Strength the Relationships of the Elders and their Families Living Abroad. Proceedings for Fall Symposium on Caring Machines: AI in Eldercare, AAAI's Fall Symposium, Association for the Advancement of Artificial Intelligence.

[6] Meishu Song, Zijiang Yang, Alice Baird, et al. 2019. Audiovisual Analysis for Recognising Frustration during Game-Play: Introducing the Multimodal Game Frustration Database. 2019 8th International Conference on Affective Computing and Intelligent Interaction (ACII), IEEE, 517-523.

[7] Menghe Zhang, Junsong Zhang, and Dong Zhang. 2019. ATVR: An Attention Training System using Multitasking and Neurofeedback on Virtual Reality Platform. 2019 IEEE International Conference on Artificial Intelligence and Virtual Reality (AIVR), IEEE, 159-1593.

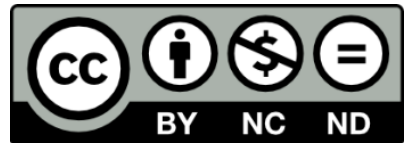

C 2021 by the authors. This work is licensed under the Creative Commons AttributionNonCommercial-NoDerivatives 4.0 International License. To view a copy of this license, visit http://creativecommons.org/licenses/by-nc-nd/4.0/ or send a letter to Creative Commons, PO Box 1866, Mountain View, CA 94042, USA. 